\title{
A practical method for determining pit depths using X-ray attenuation in EDX spectra
}

\author{
Authors: Recep Avci, Bret H. Davis, Mark L. Wolfenden, \\ Laura R. Kellerman, Kilean Lucas, Joshua Martin, \& \\ Muhammedin Deliorman
}

This is a postprint of an article that originally appeared in Corrosion Science on April 15, 2015.

Avci, Recep, Bret H. Davis, Mark L. Wolfenden, Laura R. Kellerman, Kilean Lucas, Joshua Martin, and Muhammedin Deliorman. A practical method for determining pit depths using X-ray attenuation in EDX spectra. Corrosion Science. April 2015. Pages 9-18.

http://dx.doi.org/10.1016/j.corsci.2014.12.018

Made available through Montana State University's $\underline{\text { ScholarWorks }}$

scholarworks. montana.edu 


\title{
A practical method for determining pit depths using $\mathrm{X}$-ray attenuation in EDX spectra
}

\author{
Recep Avci, Bret H. Davis, Mark L. Wolfenden, Laura R. Kellerman, Kilean Lucas, \& Joshua Martin: \\ Department of Physics, Montana State University, Bozeman, Montana \\ Muhammedin Deliorman: \\ School of Chemical Engineering and Bioengineering, Washington State University, Pullman, Washington
}

\begin{abstract}
A B S T R A C T
A practical method has been developed for rapidly determining the depth of a corrosion micro-pit from the path lengths of $\mathrm{X}$ rays passing through the walls of the pit on their way to an X-ray detector. The method takes advantage of the attenuation of the Bremsstrahlung and characteristic X-ray radiation accompanying each X-ray spectrum, and the results are verified independently using AFM and the special pit geometry surrounding MnS inclusions in 1018 carbon steel. The method has general validity and is especially valuable in those cases where the pit depth-to-width ratio is too steep to measure using the conventional methods.
\end{abstract}

\section{Introduction}

The motivation for this work arises from the need to determine the depth of the micro-pits that are initiated on carbon steel surfaces which have been exposed to anaerobic or aerobic corrosive environments [1-5]. During the course of imaging and analysis of the corrosion products and the pit initiation and development associated with MnS inclusions, very often a large number of pit formations are observed in the immediate surroundings of MnS inclusions (stringers) [6-8]. A limited number of techniques are available in the literature for determining pit depths: Liu and coworkers use pulsed thermography and high-resolution laser scanning coupled with complicated mathematical algorithms to determine pit depths which were either larger than $1 \mathrm{~mm}$ (pulsed thermography) [9] or less than $10 \mathrm{~nm}$ [10]. Davenport and coworkers used in situ synchrotron radiography and tomography to determine pit morphologies [11,12]. Zhou and coworkers employed electron tomography (using a special imaging system) coupled with ultramicrotomy to determine pit profiles [13]. Here we introduce a very practical method which uses only data from energy dispersive X-ray (EDX) microanalysis (a commonly used technique) for the rapid determination (in $\sim 5 \mathrm{~min}$ ) of pit depths in the range from a few microns to $\sim 50 \mu \mathrm{m}$ (this range is limited by the penetration depth of the characteristic $\mathrm{X}$ rays in the EDX spectra).

Surfaces were prepared by cutting and polishing 1018 carbon steel bars perpendicular to their rolling direction. The elemental composition of 1018 carbon steel, in wt.\%, is $0.14-0.16$ C, $0.6-0.9$ Mn, less than $0.004 \mathrm{P}$, less than 0.05 S and the balance Fe [14]. The stringers were very often about $1 \mu \mathrm{m}$ wide and hundreds of microns long, extending along the rolling direction of the steel bar. Fig. 1 shows the tip of one of these stringers sticking out from the bottom of a corrosion pit. The field emission scanning electron microscope (SEM) image was zoomed into the top of the MnS tip, causing an increase in the brightness of the image at the top of the pit. Comparison of the energy dispersive X-ray (EDX) spectrum obtained from the tip (Point 1 ) to the one from the top surface (Point 2) confirms that it is a MnS inclusion. Notice also that the majority of $\mathrm{X}$ rays in the energy range from 0 to $\sim 3.5 \mathrm{keV}$ are absorbed in Spectrum 1 (yellow). These stringers when exposed to an acidic environment as a result of the corrosion process start dissolving from the top and give rise abiotically to high concentrations of aggressive $\mathrm{H}_{2} \mathrm{~S}$ and $\mathrm{HS}^{-}$species inside the micro-pits, causing increases in the corrosion processes locally [14]. While pit initiation and development is a very important topic, the focus of this paper is the development of a practical method for determining the depths of the micro-pits that are often encountered on 
corroding surfaces, particularly after the corrosion products are removed. It is desirable to have some idea of the depths of these pits in relation to their local environment. These pits are often too narrow to determine their depth with standard techniques such as optical microscopy or atomic force microscopy (AFM). This is mostly because neither light nor an AFM tip can be used to probe these depths: Pits are typically too narrow and deep to get reflected light back and not wide enough for an AFM tip to fit inside the hole to probe its depth $[15,16]$. On the other hand, a wellfocused electron beam, typically a few $\mathrm{nm}$ in diameter, has little problem reaching the bottom of a pit, interacting with the material at the bottom and generating characteristic $X$ rays as well as the Bremsstrahlung radiation continuum that accompanies the characteristic $\mathrm{X}$ rays [17]. The subject of this paper is the development of a practical technique which can easily be used to obtain a rough idea of the depths of any narrow, deep holes in these samples. The presence of MnS inclusions is not necessary for the application of this method. Here we take advantage of the special geometry developed around MnS inclusions, as shown in Fig. 1, which gave us an opportunity to determine pit depth through two independent means: (1) X-ray attenuation and (2) geometrical means using high-resolution SEM images. Our previous publication [14] explains the science behind the initiation and development of these pits. Fig. 1 shows an FEM image of typical corrosion pits (left) that were initiated and developed around a MnS stringer in two weeks of exposure to D. alkanexedens (ALDC) culture [14,18]. EDX spectra (b) were taken from the MnS (yellow) and from a spot on the surface (blue) under identical conditions.

As an alternative to corrosion, artificial pits were made on a clean iron surface by means of ion milling. This was achieved by sputtering, using a focused $\mathrm{Ga}^{+}$ion beam at 12-keV impact energy focused to a $1-\mu \mathrm{m}$ size at $1-\mathrm{nA}$ current, and by rastering a $9 \times 9$ square $\mu \mathrm{m}$ area on the clean 1018 carbon surface. However, preferential etching of the iron grains with the ion beam resulted in micro-holes that were far from ideal, as shown in Fig. 2: they were not nearly as well-defined as the ones formed naturally around MnS inclusions (Fig. 1 and other figures in the manuscript). The idea behind Fig. 2 was that one could determine the pit depth accurately using atomic force microscopy (AFM) and using X-ray attenuation and compare the results. A depth measurement with AFM is also shown in Fig. 2. However, the AFM tip was too rough to probe the deep edges and corners of the pit; hence, the AFM cross-section analysis shown on the right of the AFM image gives a smoothedout bottom with a depth of about $2 \mu \mathrm{m}$ while the depth determined using X-ray attenuation, as explained below, produced a $\sim 3-\mu \mathrm{m}$ depth at the location marked as 1 in the pit. As is clearly seen in the SEM image, the depth at that location is slightly deeper than the visible bottom of the pit; however, the AFM tip could not reach these depths near the pit walls. Furthermore, the X-ray attenuation method works best if all the energy $(20 \mathrm{keV})$ of the primary electrons is dissipated within the interaction volume of the beam at the impact point. Open holes such as that shown in Fig. 2 enable the high-energy scattered electrons to escape the point of impact, causing the production of $\mathrm{X}$ rays at locations not intended for probing. We now focus our attention on electron/ material interactions.

When a high-energy electron beam (15-20 keV) focused to a diameter of a few nm impinges on a sample surface in an SEM setting, it generates X-ray radiation from an interaction volume, whose effective size for this application is assumed to be confined to $\sim 1 \mu \mathrm{m}$ [19]. The energy distribution of these $\mathrm{X}$ rays is in the form of characteristic peaks associated with the elemental composition of the specimen. These characteristic $\mathrm{X}$ rays are superimposed on a continuous background due to Bremsstrahlung radiation whose cross section is proportional to $Z^{2}$ where $Z$ is the atomic number of the material under consideration, in this case Fe and MnS inclusions [19,20].

An energetic electron, as it interacts with a material, such as steel, is dissipated through inelastic collisions with the atoms of the material. Some of these interactions give rise to the characteristic $\mathrm{X}$ rays or Auger electrons specific to the material under study as a result of the decay of the core holes in the bound states of the atoms excited by the energetic primary electrons. On the other hand, some of the primary electrons interact with the positive charges in the nuclei of these atoms, losing part of their energy and causing the emission of Bremsstrahlung radiation. Regardless of the type of $X$ rays generated, when these $X$ rays pass through a material their original intensity, $I_{o}$, decreases exponentially with increasing path length, $\ell$, through the material. The attenuation of the intensity is given by the Beer-Lambert equation (Eq. (1)) where $\mu(E)$ is the X-ray attenuation coefficient; $\frac{I}{I_{0}}$ is the ratio of the final intensity, $I$ at distance $\ell$, to the initial intensity, $I_{o}$; at $\ell=0$; and $E$ is the X-ray energy. In practice, the Beer-Lambert equation is typically written in terms of the X-ray mass attenuation coefficient, $\frac{\mu(E)}{\rho}$, where $\rho$ is the mass density of the material (Eq. (2)) [19]:

$\frac{I}{I_{o}}=\exp (-\mu(E) \ell)$

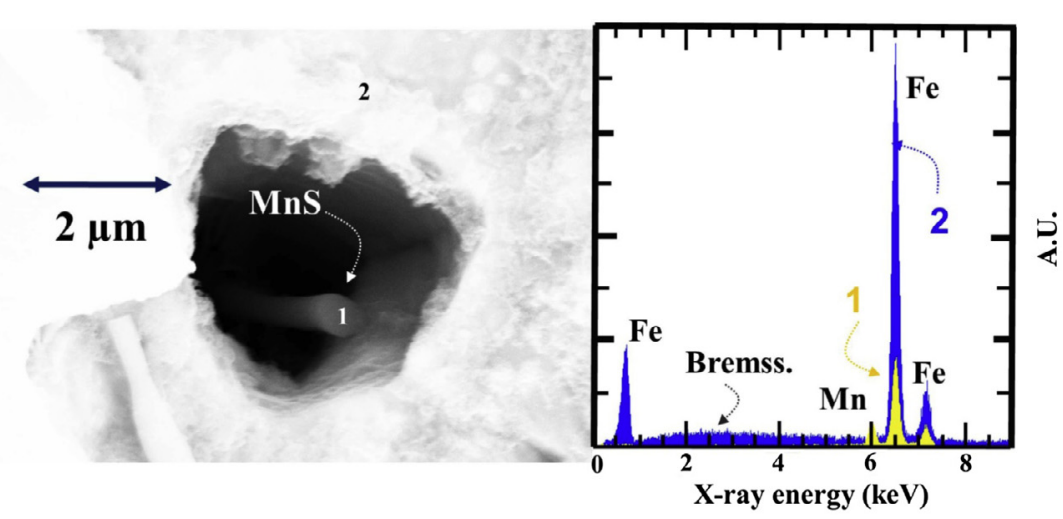

(a)

(b)

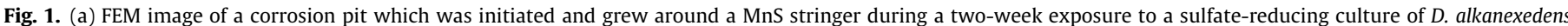

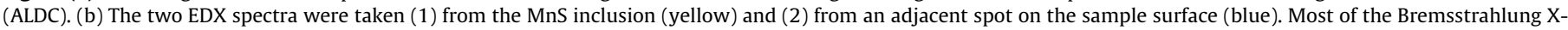
rays (marked) have been attenuated in spectra (1). 


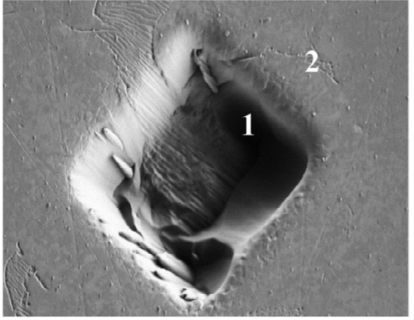

(a)

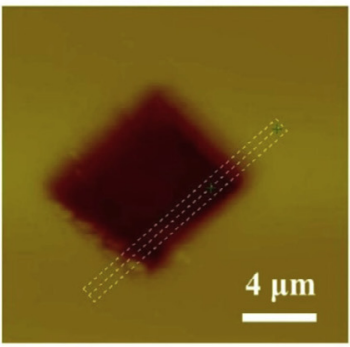

(d)

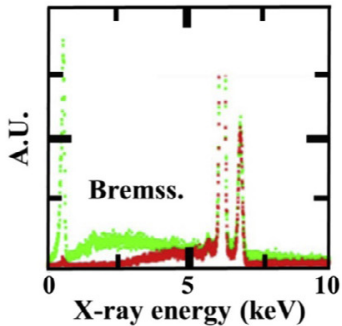

(b)

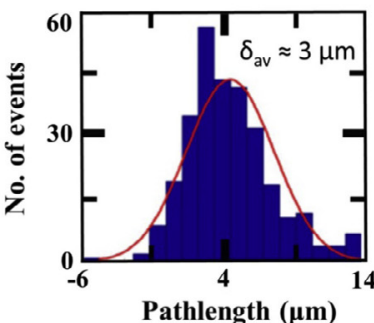

(c)

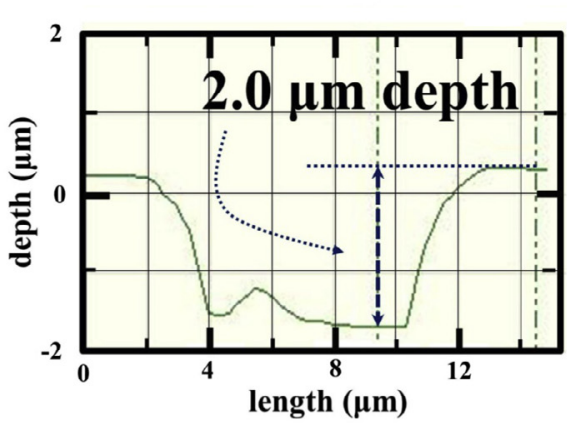

(e)

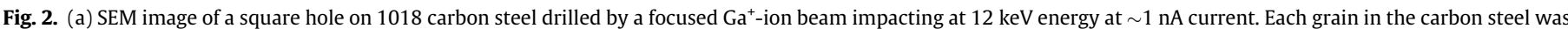

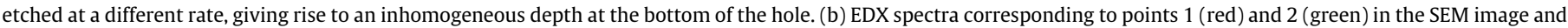

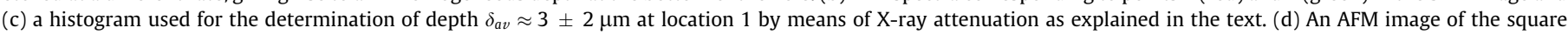

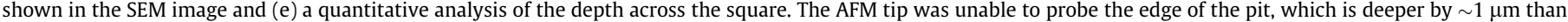
the probe can reach.

$\frac{I}{I_{0}}=\exp \left[-\left(\frac{\mu(E)}{\rho}\right) \rho \ell\right]$

The tabulated values of $\frac{\mu(E)}{\rho}$ vs. $E$ are available in NIST data bases [21] for most materials. Therefore, if the transmitted and incident X-ray intensities, $I$ and $I_{o}$, are measured as a function of X-ray energy as in EDX spectra and the material density, $\rho$, and mass attenuation coefficient, $\frac{\mu(E)}{\rho}$, vs. $E$ are known, then the path length, $\ell$, may be deduced. The mass attenuation coefficient provided by NIST is for pure iron, and so it should be noted that carbon steel is approximated to this by considering a very small fraction of carbon steel consists of carbon ( $\sim 0.15$ wt.\%.) Also, the NIST data base provides only thirteen data points in the X-ray energy range from $1 \mathrm{keV}$ to $20 \mathrm{keV}$; hence, using these data points the mass attenuation coefficient $\frac{\mu(E)}{\rho}$ as a function of continuous photon energy, E, was fitted to power law functions, as illustrated in Fig. 3.

The discontinuity in the mass attenuation coefficient at $7.112 \mathrm{keV}$, which is due to the reabsorption of $\mathrm{X}$ rays by the $\mathrm{Fe}$ $K_{\alpha, \beta}$ core excitations, requires that the power law fit be split into two functions, as shown in Eq. (3): $\frac{\mu_{1}(E)}{\rho}$ in the energy range of $1 \leqslant E<7.112 \mathrm{keV}$ and $\frac{\mu_{2}(E)}{\rho}$ in the range of $7.112<E \leqslant 20 \mathrm{keV}$, where the $A$ 's and $B$ 's are the fitting parameters.

$\frac{\mu_{1}(E)}{\rho}=A_{1} E^{-B_{1}} \quad$ for $1 \leqslant E<7.112 \mathrm{keV}$

$\frac{\mu_{2}(E)}{\rho}=A_{2} E^{-B_{2}} \quad$ for $7.112<E \leqslant 20 \mathrm{keV}$

The best-fitting parameters using the data points provided by NIST in each region of interest are tabulated in Table 1 for each formulation proposed in Equations (3) and (4), where energy, $E$, is in units of $\mathrm{keV}$ and $\frac{\mu}{\rho}$ is in units of $\mathrm{cm}^{2} / \mathrm{g}$. These fits are also plotted in Fig. 3 together with the actual data points from NIST in the energy range of interest (2-9 keV). Now the path length, $\ell$, through the steel can be easily calculated at each preselected energy point

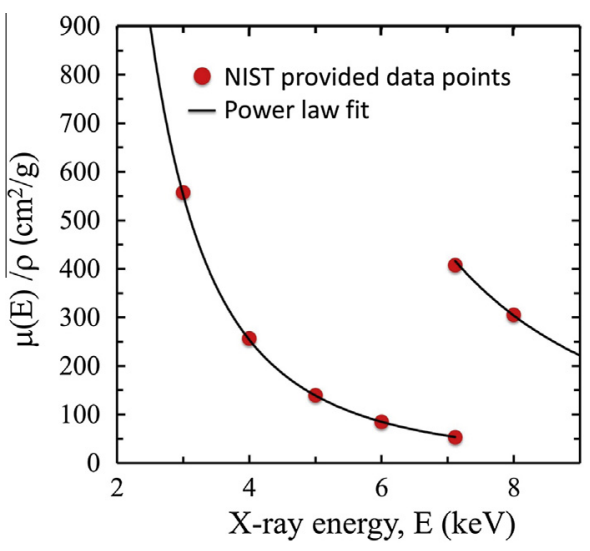

Fig. 3. Mass attenuation coefficient $\frac{\mu(E)}{\rho}$ vs. X-ray energy, $E$, in the energy range of interest for iron as tabulated by NIST (full circles) with power law fits (full lines) before and after the Fe $K_{\alpha, \beta}$ absorption edge at $\sim 7.11 \mathrm{keV}$. The sharp increase (discontinuity) in the attenuation coefficient at $\sim 7.11 \mathrm{keV}$ is due to the absorption of $\mathrm{X}$ rays at the Fe $K_{\alpha, \beta}$ absorption edge. (For interpretation of the references to color in this figure legend, the reader is referred to the web version of this article.)

Table 1

Power law function-fitting parameters $A$ and $B$ for the mass attenuation coefficient for pure $\mathrm{Fe}, \frac{\mu(E)}{\rho}=A E^{-B}$, as a function of X-ray energy, $E$, in the two X-ray ranges specified.

\begin{tabular}{llll}
\hline & A & B & X-ray range $(\mathrm{keV})$ \\
\hline$\frac{\mu_{1}(E)}{\rho}$ & $9.69 \times 10^{3}$ & 2.63 & $1 \leqslant E<7.112$ \\
$\frac{\mu_{2}(E)}{\rho}$ & $7.99 \times 10^{4}$ & 2.68 & $7.112<E \leqslant 20$ \\
\hline
\end{tabular}

between $2 \mathrm{keV}$ and $9 \mathrm{keV}$ in the EDX spectrum. Using the mass attenuation coefficient and the incident and transmitted intensities, by rearranging Eq. (1) the path length $\ell(E)$ for a given X-ray energy $E$ can be determined using Eq. (5). 
$\ell(E)=\frac{\ln \left(\frac{I_{o}}{I}\right)}{\mu(E)}$, where $\mu(E)=\left(\frac{\mu(E)}{\rho}\right) \times \rho$

It is expected to be a distribution of path lengths because of the $\mathrm{X}$-ray spectral noise and finite acceptance angle of the detector $\left( \pm 6^{\circ}\right)$. The average value (Eq. (6)) will be considered the path length of interest where

$\ell_{a v} \cong \frac{1}{N_{o}} \sum_{E_{i}} \ell\left(E_{i}\right)$

$N_{o}$ is the total number of energy points $E_{i}$ considered. In choosing the energy ranges of interest three guiding principles are followed: (1) avoiding having the characteristic $\mathrm{X}$ ray in the region of interest if the Bremsstrahlung continuum is to be used, because the elemental composition (MnS) at the bottom of a pit is not the same as that at the top (Fe); (2) making sure that there are enough X-ray counts, but not to the level that all the $\mathrm{X}$ rays are absorbed, so that enough transmitted (Bremsstrahlung or characteristic X-ray) radiation is registered in order to have meaningful $\frac{I_{o}}{I}$ ratios; and (3) if the pits are shallow and open, avoiding using high-energy $\mathrm{X}$ rays such as the characteristic Fe $K_{\alpha}$ line because high-energy electrons thus generated can escape the shallow holes, generating spurious $\mathrm{X}$ rays from unintended locations that lead to values smaller (or larger) than the actual values for $\ell_{a v}$. Basically it is important to make sure that all of the incident electrons are absorbed within the interaction volume of interest. This in fact is the case for narrow, deep pits; for these pits $\ell_{a v}$ is the same no matter which X-ray regions are used. Hence for deep and narrow pits it is more practical to use the characteristic X-rays lines such as Fe $K_{\alpha}$.

\section{Experiments}

Corrosion pits were initiated on highly polished (40-nm surface finish) [14,18] 1018 carbon steel coupons [22] in one of two ways: (1) by exposing the coupons to sulfate-reducing bacterial (SRB) cultures of $D$. alkanexedens (ALDC), a hydrocarbon degrading organism, at a $\sim 10^{6}$-cells/mL concentration under anaerobic conditions for a period of two weeks as described in our previous publication [14] and (2) by exposing the coupons in a $20-\mathrm{mL}$ vial to a culture of Marinobacter, a fuel-degrading aerobic organism, in $10 \mathrm{~mL}$ of Key West seawater at an initial cell concentration of $10^{7}$ cells/mL; the aqueous culture was then overlaid with $3 \mathrm{~mL}$ of JP5 fuel. The coupon was immersed into the aqueous phase $\sim 1 \mathrm{~cm}$ below the fuel/water interface. After seven days of exposure the coupons (1018 carbon steel and pure (99.995\%) Fe) were removed, rapidly rinsed with ultrapure $(18.0 \mathrm{M} \Omega \mathrm{cm})$ water then dried with dry nitrogen gas. Corrosion deposits were then analyzed using field emission SEM, stripped (by immersing the coupons very briefly $(\sim 1 \mathrm{~s})$ in a stripping agent, $6 \mathrm{M} \mathrm{HCl}$ and hexamethyltetramine: $3.5 \mathrm{~g} / \mathrm{L}$ ), immediately washed and dried, then inserted into the SEM for pit analysis. Details of the aerobic and suboxic corrosion experiments will be published elsewhere. These two different corrosion mechanisms will be referred to here as anaerobic and aerobic corrosion mechanisms, and the coupons subjected to these corrosive environments will be referred to with similar adjectives.

In both the anaerobic and the aerobic exposures of 1018 carbon steel, all the pits were found to have been initiated and grown around the immediate surroundings of MnS stringers $\sim 1 \mu \mathrm{m}$ in diameter and extending hundreds of microns into the coupons, perpendicular to the coupon surfaces. No pits were observed in the pure Fe except those that were already present on the surface before the exposure; occasionally we have observed deep pits of this kind. As a pit develops around a MnS stringer, it grows wider and deeper: The local chemistry and electrochemistry associated with the dissolution of $\mathrm{Fe}^{2+}$ ions inside these pits draw negative ions mostly $\mathrm{Cl}^{-}$into the pit region, and the hydrolysis of the $\mathrm{Fe}^{+2}$ (and perhaps $\mathrm{Fe}^{+3}$ ) ions acidifies the pit environment, causing the dissolution of $\mathrm{MnS}$ in the pit and giving rise to abiotic elemental S, $\mathrm{H}_{2} \mathrm{~S}$ and $\mathrm{HS}^{-}$which further fuel the corrosion process [14,23-25]. This process results in the pit configurations shown in Figs. 1 and 4 , where the tips of the MnS inclusions are sticking out of the pit bottom, establishing a good reference point for determining their depth from the surface by multiple means.

Images and spectra similar to those in Fig. 1 are shown in Fig. 4 for three different pits, labeled as Pit 1 through Pit 3. Corresponding spectra of the Bremsstrahlung radiation in the regions of interest are compared on the right sides of the field emission SEM images in two colors: The blue spectra were obtained from the top surface, while the yellow spectra were obtained from the MnS stringers visible inside the pits. In Eq. (5) the $\frac{I_{0}}{I}$ ratio corresponds to the ratio of the normalized height of the blue bar to that of the yellow bar shown in Fig. 4 at a given energy $E$ in the region of interest, avoiding the $\mathrm{S} K_{\alpha}$ primary X rays. A solid state X-ray detector, based on a $\mathrm{Si}(\mathrm{Li})$ crystal, with an X-ray acceptance area of $30 \mathrm{~mm}^{2}$ was located $\sim 28 \mathrm{~mm}$ from the sample. The mean angle of detection was $\sim 35^{\circ} \pm 6^{\circ}$ from the horizontal, resulting in a maximum solid angle of $\sim 3.8 \times 10^{-2} \mathrm{sr}$. The $\mathrm{X}$ rays transmitted

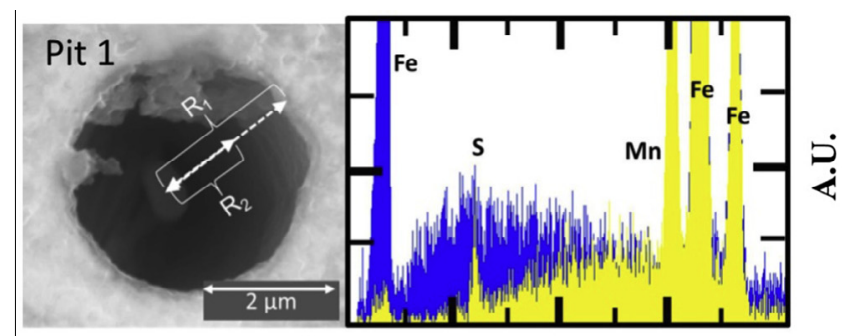

(a)

(b)

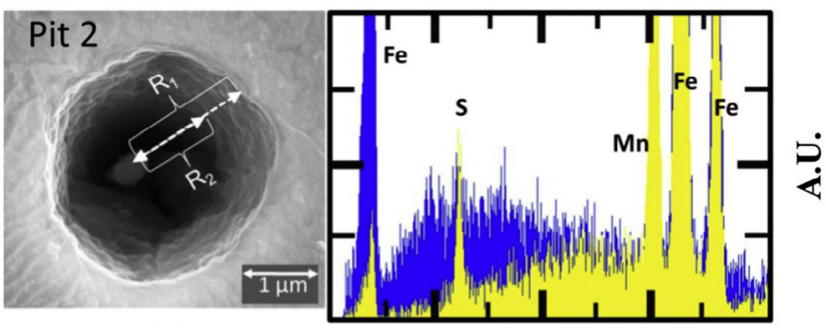

(c)

(d)

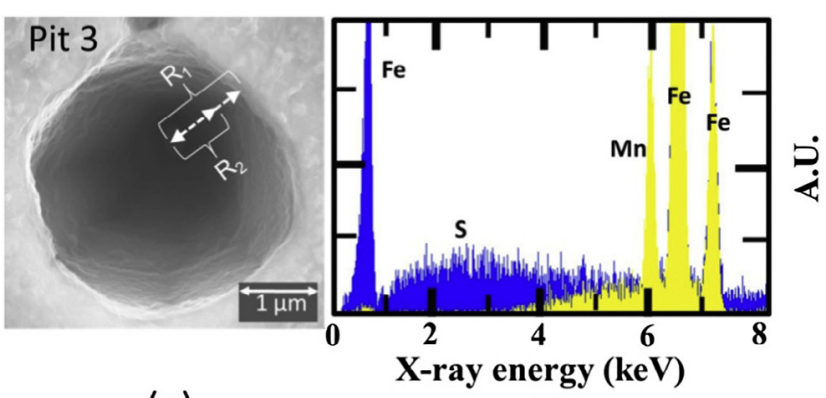

(e)

(f)

Fig. 4. Three SEM images (a, c, and e) of pits with MnS inclusions at their bottoms labeled, respectively, Pit 1, Pit 2 and Pit 3. Two superimposed EDX spectra per pit (b, $\mathrm{d}$, and $\mathrm{f}$ ), with the blue spectrum taken from the top of the pit and the yellow spectrum from the MnS at the bottom. The spectra are scaled up to emphasize the Bremsstrahlung radiation of interest and the spectral noise. For the pit radii, $R_{1}$ and $R_{2}$, see Fig. 5. Each radius is measured from an axis perpendicular to the top of the inclusion (oval shape) at the center of a pit. The determination of $R_{2}$ as described here is the largest uncertainty in the geometric measurements. 


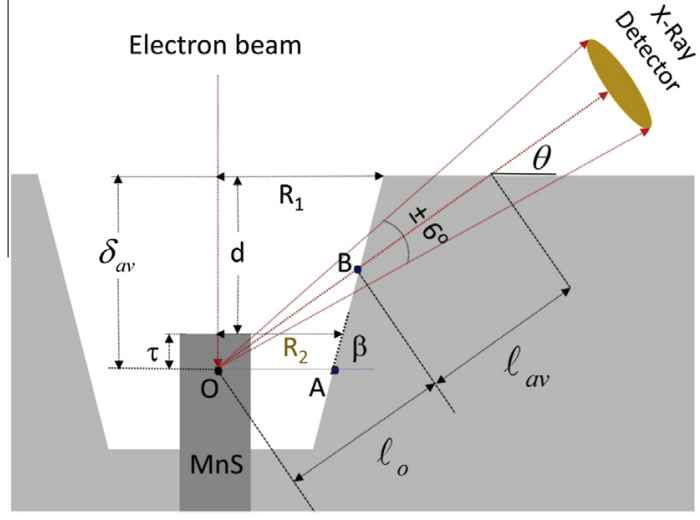

Fig. 5. Geometric representation of a pit as seen from a cross section in the plane defined by the incident electrons aimed at the center of the MnS inclusion and the exiting $\mathrm{X}$ rays pointed at the center of the $\mathrm{X}$-ray detector. Also shown are critical parameters $R_{1}, R_{2}, d, \ell_{0}, \ell_{a v}, \delta_{a v}, \theta, \beta, \tau$ and the triangle $\mathrm{OAB}$ used in calculations. The $\mathrm{X}$-ray detector is fixed at an angle of $\theta \approx 35^{\circ} \pm 6^{\circ}$ from the horizontal. Distance $\mathrm{d}$ is the depth of the MnS surface from the top of the pit. $R_{1}$ and $R_{2}$ are the horizontal distances from the axis to the inclined walls of the pit. Angle $\beta$ is the inclination angle of the pit walls. Distance $\tau \approx 1 \mu \mathrm{m}$ is assumed to be the average depth of the excitation volume from the MnS inclusion for a $20-\mathrm{keV}$ e-beam.

through a path length of $\ell$ were generated from an excitation volume with a dimension of $\sim 1 \mu \mathrm{m}$, which can be assumed to be a point source relative to the X-ray detector distance provided that the incident electrons are all confined in this region (i.e. narrow, deep pit). The primary beam was focused at the center of the MnS tips at the bottoms of the pits shown in Figs. 1 and 4 . The

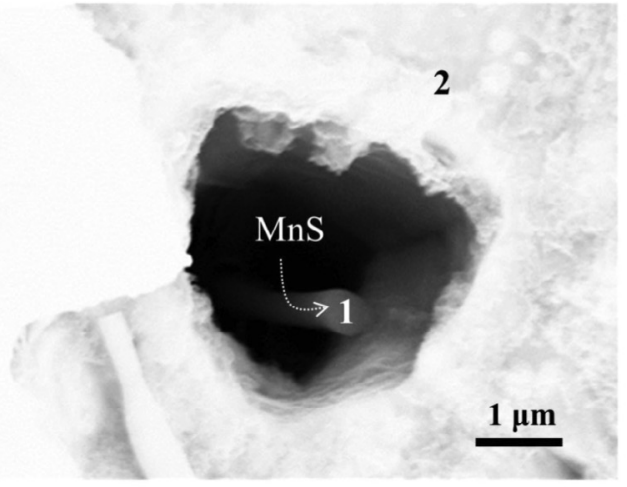

(a)

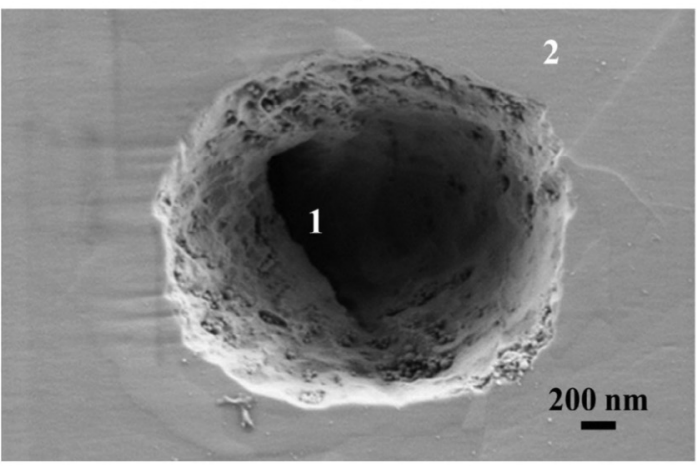

(d)

direction of the X-ray travel path projected onto the paper plane points from the center of the MnS pit to the upper right-hand corner of the field of view of the scanned SEM image, as shown in Fig. 4. The $R_{1}$ and $R_{2}$ horizontal distances between an axis perpendicular to the center of the inclusion and the walls of the pit, inclined at an angle $\beta$ (see Fig. 5) from the horizontal, are also shown in the figure. Therefore, the $\mathrm{X}$ rays exiting the interaction volume at point $O$ (assumed to be $\sim 1 \mu \mathrm{m}$ below the top of the MnS inclusion) falling within a cone shown in Fig. 5 inclined at $\theta=35^{\circ} \pm 6^{\circ}$ from the horizontal will be detected by the X-ray detector. This geometry will contribute to the distribution, though not significantly, of path lengths $\ell(E)$. Taking into account the noise in the X-ray distribution (see zoomed in EDX spectra in Fig. 4), we assume that the histogram distribution of $N$ vs. $\ell$ will have a normal (Gaussian) distribution. Examples of such distributions are shown in Fig. 6. The points in the spectra collected from the MnS (yellow in Fig. 4) represent the attenuated X rays (I), and the points in the spectra taken from a spot on top of the surface (blue in Fig. 4) represent the reference intensity $I_{0}$.

Bremsstrahlung radiation is dependent on the atomic number and density of the probed material. Hence we assume that the attenuated Bremsstrahlung represented by yellow spectra in Fig. 4 before it was absorbed by the walls is an approximation of the unattenuated Bremsstrahlung reference spectra (blue) taken from the top. The Bremsstrahlung radiation from the top is mostly generated by the Fe nuclei, while the Bremsstrahlung radiation from the top of the MnS inclusion is generated by the nuclei of $\mathrm{Mn}, \mathrm{S}$, and $\mathrm{Fe}$ (we always observe a substantial Fe signature in the EDX spectra from a typical-size $(\sim 1-\mu \mathrm{m})$ MnS inclusion). For such cases the effective attenuation coefficient, $\left(\frac{\mu}{\rho}\right)_{e f}$, as shown

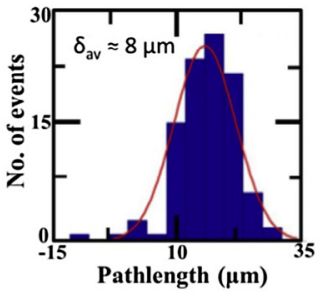

(b)

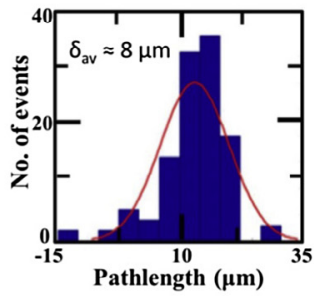

(c)

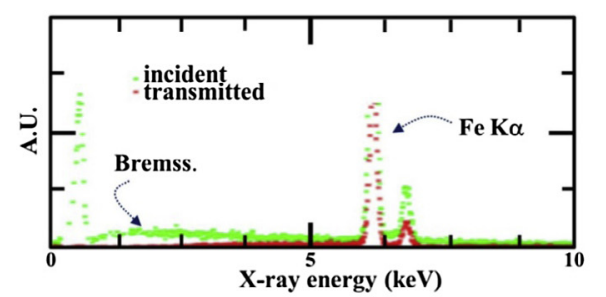

(e)

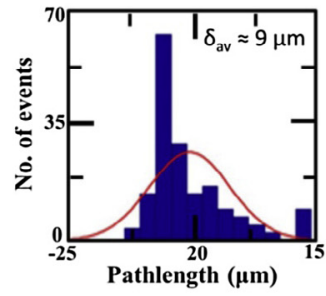

(f)

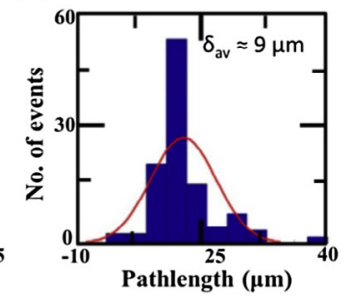

(g)

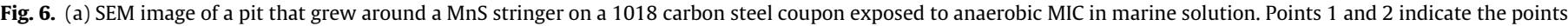

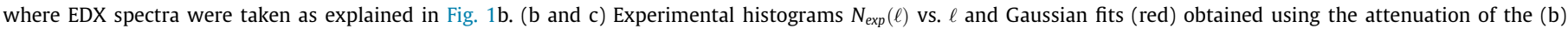

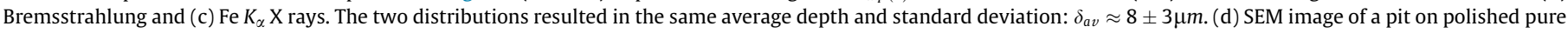

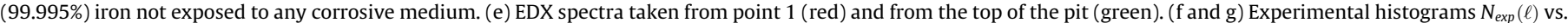

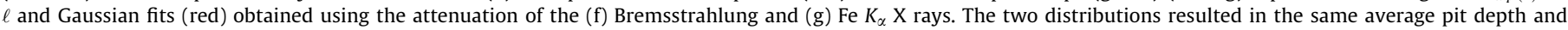
standard deviation: $\delta_{a v} \approx 9 \pm 3 \mu \mathrm{m}$. (For interpretation of the references to color in this figure legend, the reader is referred to the web version of this article.) 
in Eq. (7) is calculated from the weighted average of $\frac{\mu_{i}}{\rho_{i}}$ for each element where $\omega_{i}$ is the relative weight fraction of element $i$. In our case it is challenging to determine $\omega_{i}$ accurately because the high-energy primary electrons also probe the immediate surroundings of these thin inclusions. We assumed that $\left(\frac{\mu}{\rho}\right)$ for Fe would be an adequate representation of $\left(\frac{\mu}{\rho}\right)_{\text {ef }}$.

$\left(\frac{\mu}{\rho}\right)_{e f}=\omega_{i}\left(\frac{\mu_{i}}{\rho_{i}}\right)$

As discussed below, we tested the validity of this approximation by comparing a pair of spectra taken from the top of the surface, one from a MnS inclusion and the other from the Fe with no obstacles between the interaction volume and the X-ray detector, and found that the Bremsstrahlung profiles and intensities of the two spectra were the same within $\pm 0.2 \mu \mathrm{m}$ accuracy in the determination of the attenuation length, $\ell_{a v}$. This error is much smaller than the errors we have in the determination of $\ell_{a v}$ (from $20 \%$ to $100 \%$ ). However, as shown in Fig. 2 the $\ell_{a v}$ appears to be much closer to the independently verified value than the errors would suggest. The source of errors can be traced to the quantum noise of the X-ray statistics (see spectral noise in the EDX spectra in Fig. 4). This error is somewhat reduced in determining the depth of deep pits, as is discussed later in this work.

Comparison of the blue and yellow spectra in Fig. 4 shows attenuation of the $X$ rays is highest at the lower-energy end of the spectrum and also above $7.1 \mathrm{keV}$, as suggested by Fig. 3. The pit depth, $\delta_{a v}$, will be determined in two independent ways for these three pits only: (1) using the X-ray attenuation to determine $\ell_{a v}$ using Eq. (6) and then $\delta_{a v}$ using the relation shown in Eq. (8), where $\ell_{0}$ is the distance $\mathrm{X}$ rays travel inside the pit before encountering the walls of the pit and $\theta \approx 35^{\circ}$, and (2) using a geometric method (Eq. (9)) in which $\tau \approx 1 \mu \mathrm{m}$ and d is determined geometrically by imaging the pit from the top with no tilt and again at a tilt angle of $\gamma \approx 15^{\circ}$ from horizontal as explained below. Once our confidence in the method is established the technique will then be applied to determining the depths of four pits which will serve as examples of how this technique is expected to be used in practice.

$\delta_{a v}(\mu \mathrm{m}) \approx\left(\ell_{o}+\ell_{a v}\right) \sin \theta$

$\delta_{a v}(\mu \mathrm{m}) \approx d+\tau$

\section{Results and discussions}

\subsection{Determination of pit depth using $X$-ray attenuation}

Pits were imaged in a field emission scanning electron microscope (SEM) using a ZEISS SUPRA 55VP microscope (Carl Zeiss Microlmaging, GmbH, Gottingen) together with an energy dispersive X-ray spectrometer (EDX) from Princeton Gamma-Tech, Inc. (Rocky Hill, NJ). EDX spectra were collected for about $45 \mathrm{~s}$ at Xray energies between 0 and $15 \mathrm{keV}$ using a 20-keV primary beam energy and a working distance from the objective lens of $15 \mathrm{~mm}$. EDX spectra were analyzed using the Spirit, version 1.07.05, software provided by Princeton Gamma-Tech. As mentioned above, the X-ray detector (a $\mathrm{Si}(\mathrm{Li})$ crystal) was about $28 \mathrm{~mm}$ from the analysis point inclined $35^{\circ}$ from the horizontal with a $\pm 6^{\circ}$ acceptance angle. The X-ray detector had a circular $30-\mathrm{mm}^{2} \mathrm{X}$-ray collection area.

The MnS inclusions investigated in this study emitted a range of continuous radiation clear of substantial interference from characteristic $\mathrm{X}$ rays outside the $\mathrm{S} K_{\alpha}(2.3-\mathrm{keV}), \mathrm{Mn} K_{\alpha}(5.9-\mathrm{keV})$, Fe $K_{\alpha}$ $(6.4-\mathrm{keV})$ and $\mathrm{Fe} K_{\beta}(7.1-\mathrm{keV})$ peaks. The peak widths, depending on the particular core excitation, were below $500 \mathrm{eV}$ in the energy range of interest. The iron mass density, $\rho$, was taken as $7.87 \mathrm{~g} / \mathrm{cm}^{3}$. An appropriate Bremsstrahlung radiation energy range was chosen for each pit to avoid interference from characteristic $\mathrm{X}$ rays as well as to make sure that the transmitted Bremsstrahlung radiation stayed above the noise level once it passed through the walls of the pit. This choice was different for each pit analyzed. Both Bremsstrahlung radiation and $\mathrm{Fe} K_{\alpha} \mathrm{X}$ rays were used to determine pit depths, and the results are compared in Fig. 6. The pit depths are tabulated in Table 2. No differences in the average pit depths were observed according to whether Bremsstrahlung or characteristic $\mathrm{X}$ rays were used.

A Matlab code was written, and energy-dependent path lengths $\ell(E)$ vs. $E$ were calculated using Eq. (5). The histogram distribution $N_{\exp }(\ell)$ vs. $\ell$ was plotted for the energy regions specified. Because of the quantum noise of X-ray generation and to a lesser degree the angular variations in X-ray detection, a nonzero distribution of $\ell(E)$ is observed. This distribution was fitted with a normal (Gaussian) probability distribution, which is superimposed on experimental histograms $N_{\exp }(\ell)$ vs. $\ell$ in Figs. 2 and 6 for each pit. The average and standard deviations were calculated using standard techniques. Gaussian distribution improves with increased data points. The spread in the distribution is attributed to mostly the quantum noise in the X-ray emission distribution.

To investigate the error introduced by using the MnS inclusions and their surroundings as the sources of Bremsstrahlung radiation generated inside the pits rather than the iron matrix nuclei alone on the tops of the pits, we used a freshly polished 1018 steel surface with all the MnS inclusions either at the same level as the surface or no more than $\sim 50 \mathrm{~nm}$ (size of the particles in the polishing paste) below the surface as confirmed by AFM imaging [14,18]. We compared the Bremsstrahlung radiation intensity distribution generated from the tops of the MnS inclusions and that generated by the Fe matrix from the same surface for eight MnS inclusions. It is worth emphasizing here that for these experiments the $\mathrm{X}$ rays generated from $\mathrm{MnS}$ inclusions entered the X-ray detector without passing through any obstacle. Any differences in the intensity distributions are entirely due to differences in the material matrixes. We calculated the path lengths and their distributions using Eq. (5) for the Bremsstrahlung radiation originating from the eight MnS inclusions in combination with the two sources of incident Bremsstrahlung radiation from the Fe surface using the methods described above. This gave a total of sixteen calculated residual path lengths from MnS inclusions entirely due to material differences. The average of these residual path lengths was $\sim 0.20 \mu \mathrm{m}$. This is at least an order of magnitude smaller than the standard deviations associated with the $N_{\exp }(\ell)$ vs. $\ell$ histograms. Hence, material differences alone cannot account for the errors in the average path lengths reported here. It is safe to say that if the bottom of the pit and the top of the pit are of the same material, this might slightly improve the method described here, as demonstrated using a pit on a pure iron sample, in Fig. $6 \mathrm{~d}$.

In order to determine the pit depth from Eq. (8) we need to determine $\ell_{0}$, the distance $X$ rays travel in the vacuum inside the pit before striking the walls of the pit. In practice, if the pit is very narrow and steep then $\ell_{0} \approx 0$. This will be the case for the majority of the pits. In fact, one can deliberately aim the electron beam very near the walls and into narrow, deep holes of the pit to make sure

\section{Table 2}

Examples of X-ray energy regions for Bremsstrahlung and Fe $K_{\alpha}$ radiations used for calculating $\ell(E)$ vs. $E$ for Pits a and d shown in Fig. 6. The same results are obtained for the two regions.

\begin{tabular}{llll}
\hline & Bremsstrahlung $(\mathrm{eV})$ & Fe $K_{\alpha}(\mathrm{eV})$ & $\delta_{a v}$, Pit depth $(\mu \mathrm{m})$ \\
\hline Pit a & $4700-5200$ and 9000-9600 & $6000-6600$ & $8 \pm 3$ \\
Pit d & $3900-4600$ and 9000-9800 & $5900-6500$ & $9 \pm 3$ \\
\hline
\end{tabular}




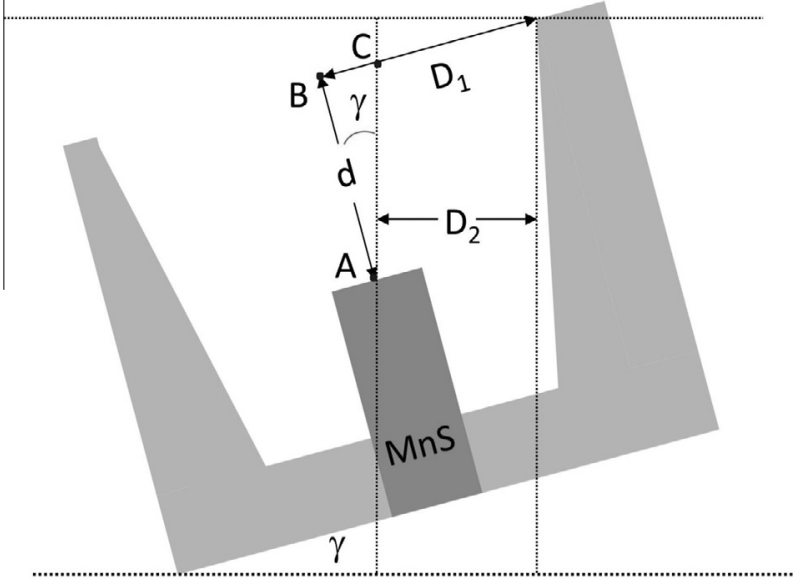

Fig. 7. Schematic of the pit shown in Fig. 5, now tilted counterclockwise by an angle of $\gamma \approx 15^{\circ}$ around an axis passing through the top of the MnS inclusions and perpendicular to the page plane in order to determine the depth, $d$. Distance $D_{1}$ is the projected distance from the center of the inclusion to the pit edge on the sample surface before it was tilted, and $D_{2}$ is the same distance after the tilt. that $\ell_{0} \approx 0$. However, in this work, we avoided this to make sure that we had a second, independent, way of verifying our depth measurements before we made use of the method. For this we had to determine the $\ell_{0}$ associated with each pit geometry. Choosing to aim the electron beam at an open target such as the top of a $\mathrm{MnS}$ inclusion will result in spurious $\mathrm{X}$ rays besides the ones generated from the points of interest. This will result in extra errors in determining the depths and comparing them with the expected values. In practice, for electron beams aimed into narrow, deep pits this situation will be avoided and results will be more accurate. Here we just want to confirm with some alternative measurement that the two results are within acceptable range of each other. We have already demonstrated this with Fig. 2 and determined that AFM and X-ray attenuation technique give us very reasonable values. This is an attempt to see similar results before we apply the techniques in real-world situations.

\subsection{Determination of pit depth using the geometric approach}

The simple geometry of the corrosion pits makes it possible to determine the approximate depth, $d$, of the top of the MnS

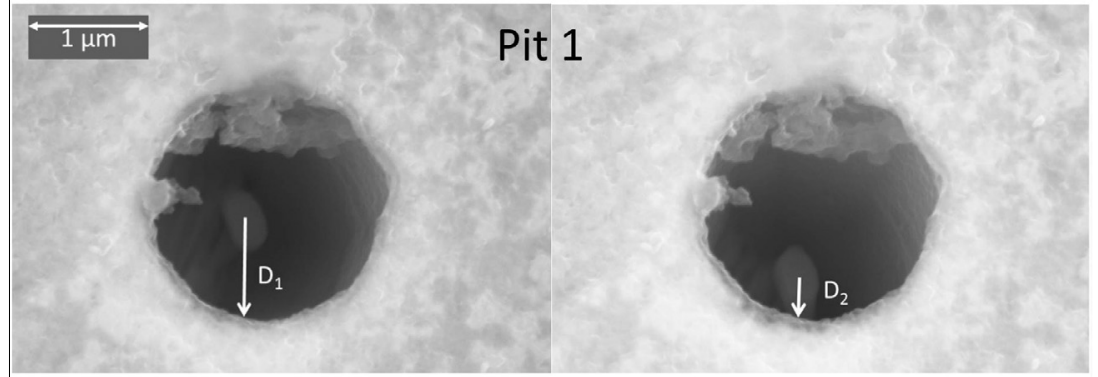

(a)

(b)

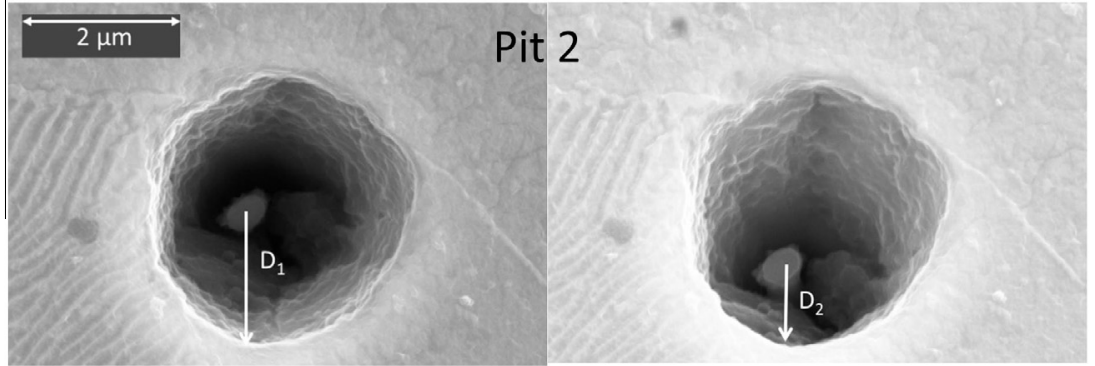

(c)

(d)

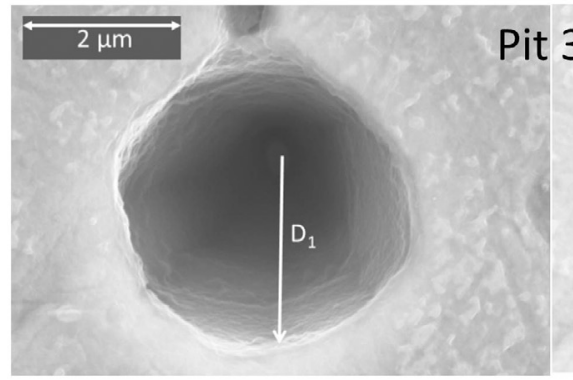

(e)

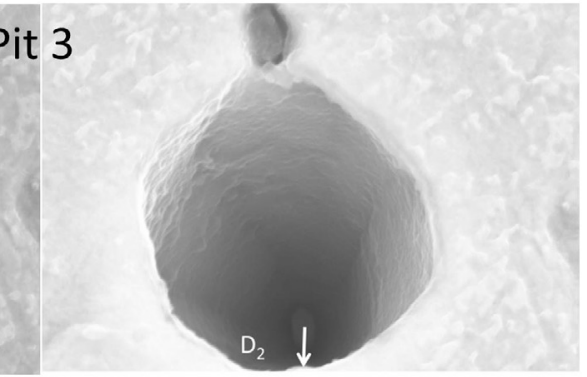

(f)

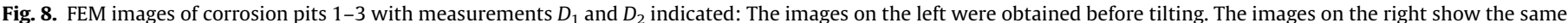

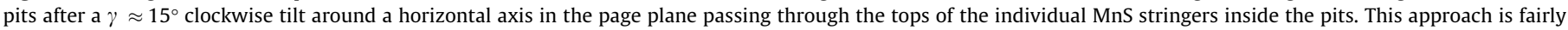
trivial and introduces minimal error into determining the $D$ 's for shallow pits. 
inclusion from the surface as well as the parameter $\ell_{0}$ as marked in the schematic representation of a pit cross section in Fig. 5. The SEM images in Fig. 4 show the determination of the critical parameters $R_{1}$ and $R_{2}$, which are also marked in Fig. 5. These critical distances can be measured directly from the image contrast differences. For these measurements it is important that the sample not be tilted and that the top views of the pictures are used. These values are recorded in Table 3. The measurement of $R_{2}$ in this approach is a bit ambiguous, and substantial (30-50\%) uncertainty can be introduced in reading $R_{2}$ and hence in determining $\beta$ and $\ell_{0}$.

Applying the law of sines to the OAB triangle in Fig. 5, the value of $\ell_{0}$ can be obtained immediately from Eq. (10) where $\theta \approx 35^{\circ}, \tau \approx 1 \mu m, \beta \approx \tan ^{-1}\left(\frac{R_{1}-R_{2}}{d}\right)$ and depth $d$ can be measured directly as explained below. From this information the pit depths, $\delta_{a v}(\mu \mathrm{m})$, can be calculated using Eq. (8), where $\ell_{a v}$ are obtained from the X-ray attenuation data and are listed in Table 3.

$\ell_{o}=\frac{\sin (180-\beta)}{\sin (\beta-\theta)}\left(R_{2}-\frac{\tau}{\tan \beta}\right)$

As mentioned above, the pit depths, $\delta_{a v} \approx d+\tau$, can also be measured independently by geometrical means: Since we assume $\tau \approx 1 \mu \mathrm{m}$ we just need to measure the distance $\mathrm{d}$ marked in Fig. 5 . This measurement is done simply by taking two critical distance measurements, $D_{1}$ and $D_{2}$, marked schematically in Fig. 7 and shown in a FEM image in Fig. 8 for each pit. This is a simple measurement between two well-defined geometries. First, $D_{1}$ is measured (see Fig. 8) when the sample surface is not tilted; second, $D_{2}$ is measured when the sample is tilted by an angle of $\gamma \approx 15^{\circ}$ around a horizontal axis perpendicular to the X-ray emission plane (paper plane) passing through the top of the MnS inclusion in the pit. This is achieved by maintaining the SEM focus on the top of the MnS inclusion. This is done for each pit. The images and the critical distances are also recorded in Table 3.

From these measurements it is trivial to determine depth $d$ using a simple geometric relation and applying the law of sines to the triangle defined by $A B C$ in Fig. 7: One obtains Eq. (11) where angle $\gamma \approx 15^{\circ}$. This, then, completes the direct (geometric) measurement of the depth: $\delta_{a v} \approx d+\tau$.

$d=\frac{D_{1}-D_{2}}{\cos \gamma \tan \gamma}$

The results from the two independent methods (geometry and $\mathrm{X}$-ray attenuation) of determining pit depths are all summarized in Table 3.

The comparisons indicate the results from the geometric method and the X-ray attenuation method agree marginally at best. This was expected. The values obtained using the geometric

Table 3

Determination of pit depths using the geometric approach and the X-ray attenuation approach. For the definitions of these parameters refer to Figs. 5, 7 and 8 .

\begin{tabular}{llll}
\hline & Pit 1 & Pit 2 & Pit 3 \\
\hline$R_{1}(\mu \mathrm{m}), R_{2}(\mu \mathrm{m})$ & $1.3,0.7$ & $2.0,1.1$ & $1.3,0.6$ \\
$D_{1}(\mu \mathrm{m}), D_{2}(\mu \mathrm{m})$ & $0.9,0.4$ & $1.6,1.0$ & $2.3,0.5$ \\
$d(\mu \mathrm{m})$ & 1.6 & 2.1 & 6.7 \\
$\ell_{0}, \ell_{a v}(\mu \mathrm{m})$ & $0.6,3.3$ & $1.1,2.9$ & $0.7,7.2$ \\
$\delta_{a v}($ Geometry $) \approx d+\tau(\mu \mathrm{m})$ & 2.6 & 3.1 & 7.7 \\
$\delta_{a v}($ X-ray $) \approx\left(\ell_{0}+\ell_{a v}\right) \sin \theta(\mu \mathrm{m})$ & $2.2 \pm 1.6$ & $2.3 \pm 1.6$ & $4.6 \pm 2.0$ \\
\hline
\end{tabular}

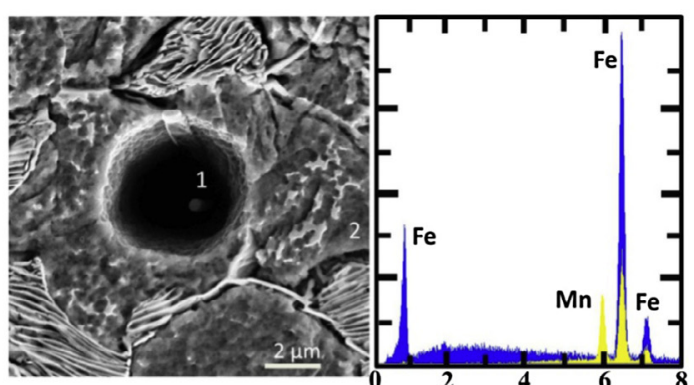

(a)

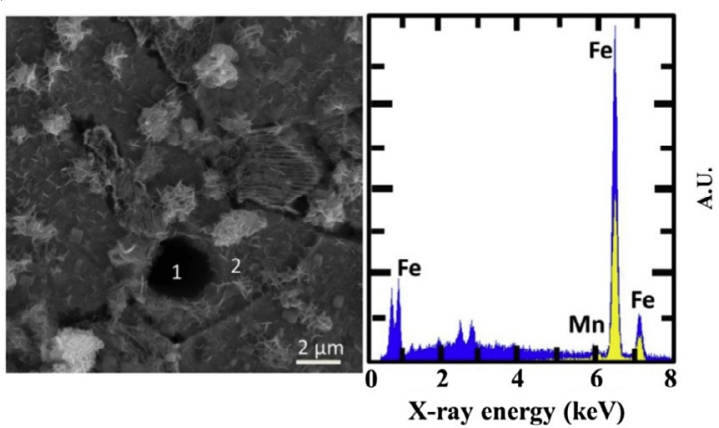

(e) (f)

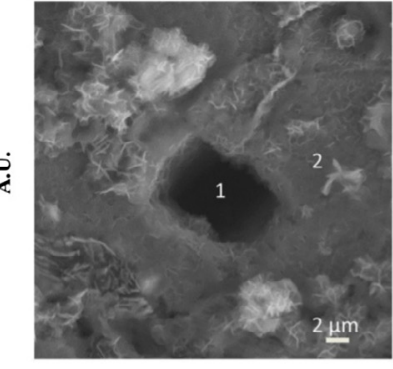

(c)

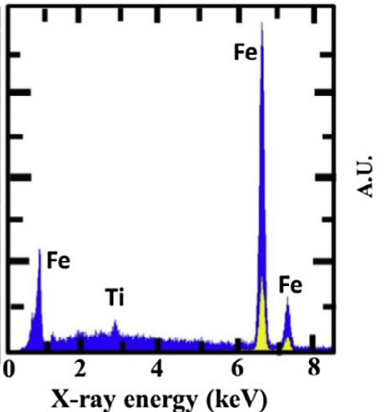

(d)

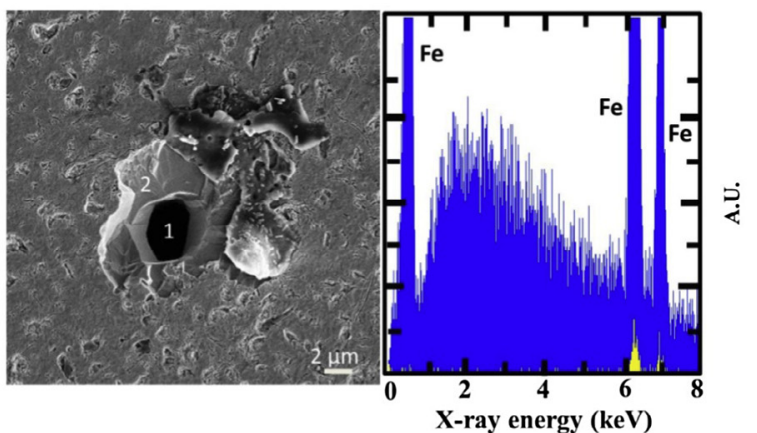

(g)

(h)

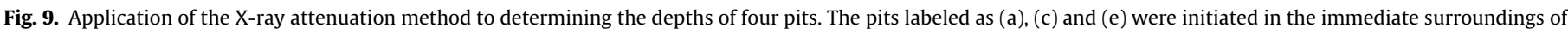

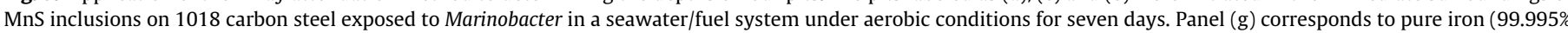

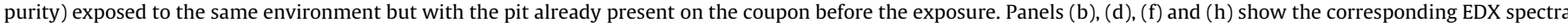
taken from the points marked as 1 (yellow) and 2 (blue). They show the attenuation of the X rays used in determining the pit depths tabulated in Table 4. 
methods are more likely close to the correct depths. The reason for this is electron scattering: The tip of a MnS inclusion creates conditions for the incident electron beam to scatter in the pit, generating $X$ rays not necessarily originating from the interaction volume localized on the MnS tip. We have seen this on many occasions. For example, in Fig. $6 \mathrm{~d}$ if the electron beam were aimed at an open area of the pit on one of the side walls the results would not have represented a true depth. X-ray attenuation, as the name suggests, requires the total absorption of the incident electrons within an interaction volume and the subsequent transmission of the $\mathrm{X}$ rays transmitted through the walls of the material on their way to the detector. In this sense, the results shown in Table 3 are fairly satisfactory. A similar comparison in Fig. 2 gave a reasonable agreement, but the beam was aimed into a narrow hole. In what follows we present the application of this technique to determining the depths of a number of pits on Fe surfaces. This approach provides accurate results rapidly, in about $5 \mathrm{~min}$, because full attenuation of incident $\mathrm{X}$-rays within deep and narrow pits is achieved.

\subsection{Application of the technique}

In Fig. 9 we present four pits on Fe surfaces whose depths we are interested in determining. The pits labeled as a, c and e due to aerobic corrosion is similar to the pits developed during anaerobic corrosion discussed above, each originated in and grew from the immediate surroundings of MnS stringers. They developed during the seven-day aerobic corrosion of coupons exposed to a fuel/ seawater system inoculated with Marinobacter described above. Pit $g$ was observed on the surface of a pure (99.995\%) iron coupon exposed to the same environment for the same length of time. However, this pit was not initiated during this period, it was already present on the surface much like the one shown in Fig. 6c; in fact, there were no pits on the pure Fe surface except those that were already on the surface and those that were initiated in the immediate surroundings of inclusions, typically some sort of Si-based inclusion. Because these pits are narrow and deep, it is a very good approximation to assume $\ell_{0} \approx 0$, and the average pit depth can simply be determined from Eq. (12).

$\delta_{a v}(\mu m) \approx \ell_{a v} \sin \theta$

All we need to do is to determine $\ell_{a v}$ using the X-ray attenuation technique. This is what is shown in Table 4 .

We are confident that the average values shown in Table 4 are a fair representation of the pit depths. The error is due to X-ray statistics associated with the generation of $X$ rays. In Table 4 the attenuation of Fe $K_{\alpha} \mathrm{X}$-rays centered at $\sim 6.4 \mathrm{keV}$ is used. However, determination of $\ell_{a v}$ by means of attenuation of the Bremsstrahlung radiation gave almost identical results, except for pit a, where $\ell_{a v} \pm \Delta \ell=10 \pm 3 \mu \mathrm{m}$. This is not entirely surprising because of the excess MnS presence in the pit (see EDX in Fig. 9a). Overall, these results are fairly satisfactory. How else could we have known of the presence of the $\sim 41-\mu \mathrm{m}$ pit whose radius is less than $2 \mu \mathrm{m}$ on the pure Fe surface shown in Fig. $9 \mathrm{~g}$ ?

Table 4

Determination of pit depths associated with Fig. 9. Error $\Delta \delta$ is calculated using $\Delta \delta=\Delta \ell \sin \theta+\ell_{a v} \cos \theta \Delta \theta$, where $\Delta \theta \approx 0.05$ radians and $\theta=35^{\circ}$.

\begin{tabular}{lllll}
\hline & Pit a & Pit c & Pit e & Pit g \\
\hline $\begin{array}{c}\text { X-ray energy } \\
\text { regions }(\mathrm{eV})\end{array}$ & $6000-6600$ & $6000-6500$ & $6000-6600$ & $6000-6500$ \\
$\ell_{a v} \pm \Delta \ell(\mu \mathrm{m})$ & $15 \pm 7$ & $25 \pm 5$ & $12 \pm 4$ & $71 \pm 12$ \\
$\delta_{a v} \approx \ell_{a v} \sin \theta \pm \Delta \delta(\mu \mathrm{m})$ & $8 \pm 4$ & $14 \pm 4$ & $7 \pm 2$ & $41 \pm 10$ \\
\hline
\end{tabular}

\section{Conclusions}

We have demonstrated that the attenuation of $X$ rays can be utilized to determine the depths of deep, narrow pits on material surfaces. We applied this technique to corrosion pits initiated and developed around MnS stringers oriented perpendicular to a 1018 carbon steel surface cut and polished perpendicular to the rolling direction of the steel bars. The value of the technique does not lie in its accuracy but in its practicality and uniqueness. While a material surface is being analyzed using SEM X-ray microanalysis, narrow, deep pits or holes are often encountered. It is desirable to have some idea of the depth of these pits or holes at the time of analysis. The technique reported here fills this niche need. In developing this technique we took advantage of the special geometry of corrosion initiation and development around a MnS inclusion so that two independent ways can be used to determine the same depth. For this purpose we used the Bremsstrahlung continuum and its attenuation as it passed through the material on its way to an X-ray detector. If, however, the composition at the bottom and at the top of the pit are the same matrix, then one can use the areas, $I$ and $I_{0}$, under the characteristic X-ray peaks as demonstrated for pure Fe and for carbon steel and simply use Eq. (13) to determine

$\ell_{a v} \approx \ell(E)=\frac{\ln \left(\frac{I_{o}}{I}\right)}{\left(\frac{\mu(E)}{\rho}\right) \times \rho}$

the depth of the pit roughly by $\delta_{a v} \approx \ell_{a v} \sin \theta$, where $\frac{I_{o}}{I}$ is the ratio of the area under a characteristic X-ray peak such as Fe $K_{\alpha}$ taken from the top of the pit to that of one taken from the bottom and $\theta$ is the angle the $\mathrm{X}$ rays make with the horizontal on their way to the X-ray detector.

Our comparison results from two different methods presented in Table 3 are reasonable, considering that these pits are very shallow and the tops of the MnS inclusions are surrounded with open regions for high-energy electrons to scatter, bounce around and perhaps even escape the pit region. However, if the pits are narrow and deep and the energetic electrons directed at the bottom of the pit are all absorbed within the interaction volume of the electrons, then the results will be fairly accurate as in Table 4 in spite of the uncertainties introduced by the X-ray production statistics. Accuracy can be improved somewhat by reducing the noise in the X-ray statistics to produce a better signal-to-noise ratio. However, the average values of the pit depths determined by X-ray attenuation are a fairly good representation of the true values in spite of the large variations in the X-ray counting statistics. This approach produces results rapidly and accurately. It only takes about 5 min to determine the depth of a pit; this includes the time to acquire two EDX spectra and to insert the data into the Matlab software.

\section{Acknowledgements}

This work is supported by ONR Multidisciplinary University Research Initiative (MURI) grant N00014-10-1-0946. The work is also supported in part by Montana State University funds supporting the Imaging and Chemical Analysis Laboratory (ICAL). The authors would like to acknowledge L. Loetterle for her contribution to the microbiological aspects of this work, members of Prof. J. Suflita's group at the University of Oklahoma for their help in providing and advising on the growth of ALDC cultures, and Prof. I. Beech of University of Oklahoma for providing us with the Marinobacter cultures. The help of N. Equall of ICAL in SEM analysis is greatly appreciated. 


\section{References}

[1] I.B. Beech, J.A. Sunner, Sulphate-reducing bacteria and their role in corrosion of ferrous materials, in: L.L. Barton, W.A. Hamilton (Eds.), Sulphate-Reducing Bacteria: Environmental and Engineered Systems, Cambridge University Press, 2007, pp. 459-482.

[2] J.Z. Duan, S.R. Wu, X.J. Zhang, G.Q. Huang, M. Du, B.R. Hou, Corrosion of carbon steel influenced by anaerobic biofilm in natural seawater, Electrochim. Acta 54 (2008) 22-28.

[3] D. Enning, J. Garrelfs, Corrosion of iron by sulfate-reducing bacteria: new views of an old problem, Appl. Environ. Microb. 80 (2014) 1226-1236.

[4] A. Valor, F. Caleyo, L. Alfonso, D. Rivas, J.M. Hallen, Stochastic modeling of pitting corrosion: a new model for initiation and growth of multiple corrosion pits, Corros. Sci. 49 (2007) 559-579.

[5] G.T. Burstein, C. Liu, R.M. Souto, S.P. Vines, Origins of pitting corrosion, Corros. Eng. Sci. Technol. 39 (2004) 25-30.

[6] B. Lin, R.G. Hu, C.Q. Ye, Y. Li, C.J. Lin, A study on the initiation of pitting corrosion in carbon steel in chloride-containing media using scanning electrochemical probes, Electrochim. Acta 55 (2010) 6542-6545.

[7] M.P. Ryan, D.E. Williams, R.J. Chater, B.M. Hutton, D.S. McPhail, Why stainless steel corrodes, Nature 415 (2002) 770-774.

[8] P. Schmuki, H. Hildebrand, A. Friedrich, S. Virtanen, The composition of the boundary region of $\mathrm{MnS}$ inclusions in stainless steel and its relevance in triggering pitting corrosion, Corros. Sci. 47 (2005) 1239-1250.

[9] Z. Liu, M. Genest, D. Krys, Processing thermography images for pitting corrosion quantification on small diameter ductile iron pipe, NDT\&E Int. 47 (2012) 105-115.

[10] W. Wu, Z. Liu, D. Krys, Improving laser image resolution for pitting corrosion measurement using Markov random field method, Automat. Constr. 21 (2012) $172-183$.

[11] S.M. Ghahari, A.J. Davenport, T. Rayment, T. Suter, J.P. Tinnes, C. Padovani, J.A. Hammons, M. Stampanoni, F. Marone, R. Mokso, In situ synchrotron X-ray micro-tomography study of pitting corrosion in stainless steel, Corros. Sci. 53 (2011) 2684-2687.

[12] S.M. Ghahari, D.P. Krouse, N.J. Laycock, T. Rayment, C. Padovani, T. Suter, R. Mokso, F. Marone, M. Stampanoni, M. Monir, A.J. Davenport, Pitting corrosion of stainless steel: measuring and modelling pit propagation in support of damage prediction for radioactive waste containers, Corros. Eng. Sci. Technol. 46 (2011) 205-211.

[13] X. Zhou, C. Luo, T. Hashimoto, A.E. Hughes, G.E. Thompson, Study of localized corrosion in AA2024 aluminium alloy using electron tomography, Corros. Sci. 58 (2012) 299-306.

[14] R. Avci, B.H. Davis, M.L. Wolfenden, I.B. Beech, K. Lucas, D. Paul, Mechanism of MnS-mediated pit initiation and propagation in carbon steel in an anaerobic sulfidogenic media, Corros. Sci. 76 (2013) 267-274.

[15] M.R. VanLandingham, T.F. Juliano, M.J. Hagon, Measuring tip shape for instrumented indentation using atomic force microscopy, Meas. Sci. Technol. 16 (2005) 2173-2185.

[16] S.H. Wang, S.L. Tan, G. Xu, K. Koyama, Measurement of deep groove structures using a self-fabricated long tip in a large range metrological atomic force microscope, Meas. Sci. Technol. 22 (2011).

[17] J. Goldstein, Scanning Electron Microscopy and X-ray Microanalysis, third ed. Kluwer Academic/Plenum Publishers, New York, 2003.

[18] B.H. Davis, M.Sc. Thesis: Anaerobic Pitting Corrosion of Carbon Steel in Marine Sulfidogenic Environment, Physics Department, Montana State University, 2013, pp. 156.

[19] J.I. Goldstein, D.E. Newbury, P. Echlin, D.C. Joy, A.D. Romig Jr., C.E. Lyman, C. Fiori, E. Lifshin, Scanning Electron Microscopy and X-Ray Microanalysis: A Text for Biologist, Material Scientist, and Geologist, second ed., Plenum Press, New York, 1992.

[20] H.W. Koch, J.W. Motz, Bremsstrahlung cross-section formulas and related data, Rev. Mod. Phys. 31 (1959) 920-955.

[21] J.H.S. Hubbell, S.M. Seltzer, Tables of X-ray mass attenuation coefficients and mass energy-absorption coefficients from $1 \mathrm{keV}$ to $20 \mathrm{MeV}$ for elements $Z=1$ to 92 and 48 additional substances of dosimetric interest*, NIST (2004).

[22] ASTM Standard A29/A29M - 12, 1957 (2012), Standard Specification for Steel Bars, Carbon and Alloy, Hot-Wrought, General Requirements for, ASTM International, West Conshohocken, PA, 2012.

[23] G.S. Eklund, Initiation of pitting at sulfide inclusions in stainless-steel, J Electrochem. Soc. 121 (1974) 467-473.

[24] G. Wranglen, Review article on influence of sulphide inclusions on corrodibility of Fe and steel, Corros. Sci. 9 (1969) 585-592.

[25] G. Wranglen, Pitting and sulfide inclusions in steel, Corros. Sci. 14 (1974) 331 349. 